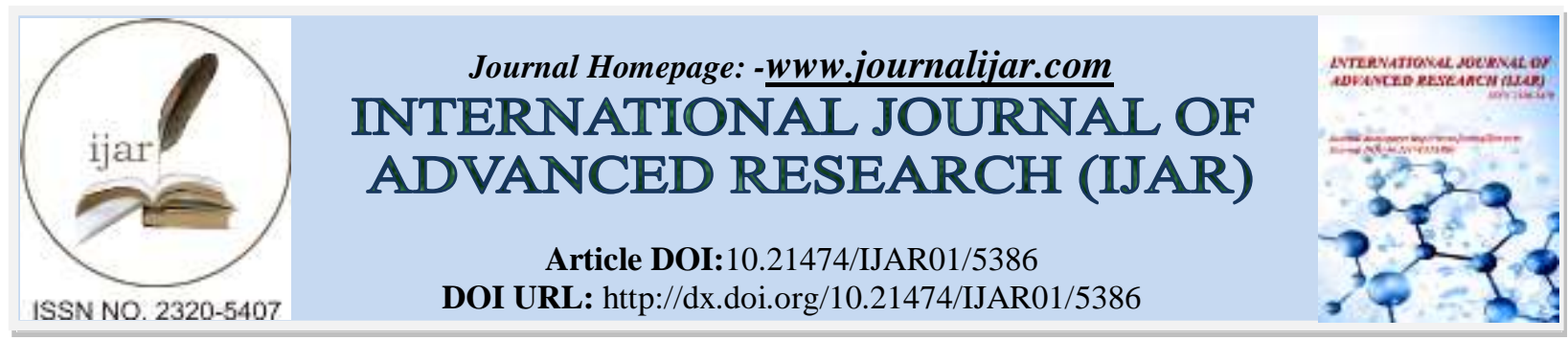

RESEARCH ARTICLE

\title{
MICROWAVE ASSISTED ONE POT SYNTHESIS OF 5-UNSUBSTITUTED-3,4-DIHYDROPYRIMIDIN- 2(1H)-ONES UNDER SOLVENT FREE CONDITION:BIGINELLI REACTION.
}

\author{
Monika Gupta ${ }^{1}$ and Anil Mishra ${ }^{2}$. \\ 1. Department of Chemistry, BabuBanarsi Das University Lucknow 226028, India. \\ 2. Department of Chemistry, University of Lucknow, Lucknow 226007,India
}

\section{Manuscript Info}

…........................

Manuscript History

Received: 10 July 2017

Final Accepted: 12 August 2017

Published: September 2017

\begin{abstract}
3,4-dihydro-6-(2,4-dihydroxyphenyl)-4-phenylpyrimidin-2(1H)-one

(4)has been synthesized by the condensation of 2,4dihydroxybenzaldehyde, acetophenone and urea with a catalytic amount of $\mathrm{ZnI}_{2}$ under microwave irradiation. In this paper the synthesis, mass spectral analysis and anti inflammatory activities of (3,4-dihydro-6-(2-hydroxyphenyl)-4-phenylpyrimidin-2(1H)-one (1) 6-(4-(dimethylamino)phenyl)-3,4-dihydro-4-phenylpyrimidin-2(1H)one (2), 3,4-dihydro-6-(3-hydroxyphenyl)-4-phenylpyrimidin-2(1H)one (3), 3,4-dihydro-6-(2,4-dihydroxyphenyl)-4-phenylpyrimidin2(1H)-one (4), 6-(3-ethoxy-2-hydroxyphenyl)-3,4-dihydro-4phenylpyrimidin-2(1H)-one (5), 3,4-dihydro-6-(4-nitrophenyl)-4phenylpyrimidin-2(1H)-one (6), 6-(2,3-diethoxyphenyl)-3,4dihydro-4phenylpyrimidin-2(1H)-one (7), 3,4-dihydro-6-(2-hydroxyphenyl)-4phenylpyrimidin-2(1H)-thione(8), 6-(4-(dimethylamino)phenyl)-3,4dihydro-4-phenylpyrimidin-2(1H)-thione (9), 3,4-dihydro-6(2,4-dihydroxyphenyl)-4-phenylpyrimidin-2(1H)-thione $\quad(\mathbf{1 0}), \quad 3,4-$ dihydro-6-(4-nitrophenyl)-4-phenylpyrimidin-2(1H)-thione(11), 6-(2,3diethoxyphenyl)-3,4-dihydro-4-phenylpyrimidin-2(1H)-thione (12) are beingreported.
\end{abstract}

Copy Right, IJAR, 2017,. All rights reserved.

\section{Introduction:-}

BIGINELLI REACTION IS A SIMPLE ONE POT CONDENSATION OF AN ALDEHYDE, KETOESTER, UREA IN A SOLVENT SUCH AS ETHANOL USING A STRONGLY ACIDIC CATALYST, THAT IS, HYDROCHLORIC ACID TO PRODUCE 3,4DIHYDROPYRIMIDIN-2-(1H)-ONES, WHICH WAS REPORTED BY BIGINELLI ${ }^{1}$. HOWEVER THE YIELD OF PRODUCTS WERE VERY LOW (J20-50\%). FROM THEN ON, MANY NEW TECHNIQUES, SUCH AS MICROWAVE ASSISTED SYNTHESIS TECHNIQUES, IONIC LIQUIDS ULTRASOUND IRRADIATION, SOLVENT-FREE TECHNIQUES AND MUCH NEW CATALYST, SUCH AS INBR 3 , ZRCL 4 , BICL 3 ETC, WERE USED TO IMPROVE THIS TRANSFORMATION. IN SPITE OF THEIR POTENTIAL UTILITY, MANY OF THESE METHODS INVOLVE EXPENSIVE REAGENTS, STRONGLY ACIDIC CONDITIONS, LONG REACTION TIMES, HIGH TEMPERATURE AND STIOCHIOMETRIC AMOUNTS OF CATALYSTS AND UNSATISFACTORY YIELDS. THE FIRST BIGINELLI LIKE REACTION WAS CONDUCTED IN $\mathrm{CH}_{3} \mathrm{CN}$ BY USING ALDEHYDES, KETONES, AND UREA AS SUBSTRATES AND FECL $3.6 \mathrm{H}_{2} \mathrm{O}$ AND TMSCL AS CATALYSTS, WHICH REMARKABLY BROADENED THE BIGINELLI REACTION ${ }^{2}$. HOWEVER, OF SUFFERED FROM ITS DRAWBACKS, ESPECIALLY THE USE OF HIGHLY TOXIC ORGANIC SOLVENT, LONG REACTION TIME (12H) AND STIOCHIOMETRICTMSCL. MANY CATALYSTS OR PROMOTERS, SUCH AS CH $\mathrm{COOH}_{3}$ 
$\mathrm{FE}_{2}\left(\mathrm{SO}_{4}\right)_{3}, \mathrm{MGSO}_{4}, \mathrm{HCL}, \mathrm{H}_{2} \mathrm{SO}_{4}$, ETC, WERE USED TO EXPLORE THE REACTION UNDER SOLVENT-FREE, MICROWAVE ASSISTED CONDITIONS. THESE RESULTS SUGGESTS THAT MOST OF THE LEWIS ACID AND BRONSTED ACIDS COULD PROMOTE THE REACTION, BUT THE YIELDS ARE NOT SO HIGH ${ }^{3}$.IN COMPARISON WITH OTHER CATALYSTS, THE USE OF 1.5 MMOL OF ZNI $_{2}$ COULD MAKE THE YIELD REACH $48 \%$ UNDER MICROWAVE IRRADIATION ${ }^{4}$. THE REASON FOR ZNI 2 BEING THE BEST CATALYST MAY BE STRONGLY ACIDIC CHARACTER ${ }^{5}$. IN ORDER TO EXAMINE THE SUBSTRATE SCOPE OF THIS BIGINELLI-LIKE REACTION, VARIOUS AROMATIC ALDEHYDES WITH DIFFERENT SUBSTITUENT USING ZNI 2 WERE USED UNDER THE OPTIMIZED REACTION CONDITION TO SYNTHESIZE A SERIES OF 5-unsubstituted-3,4dihydropyrimidin-2(1H)-ones.<smiles>[R]c1c(C)ccc(C=O)c1Br</smiles>

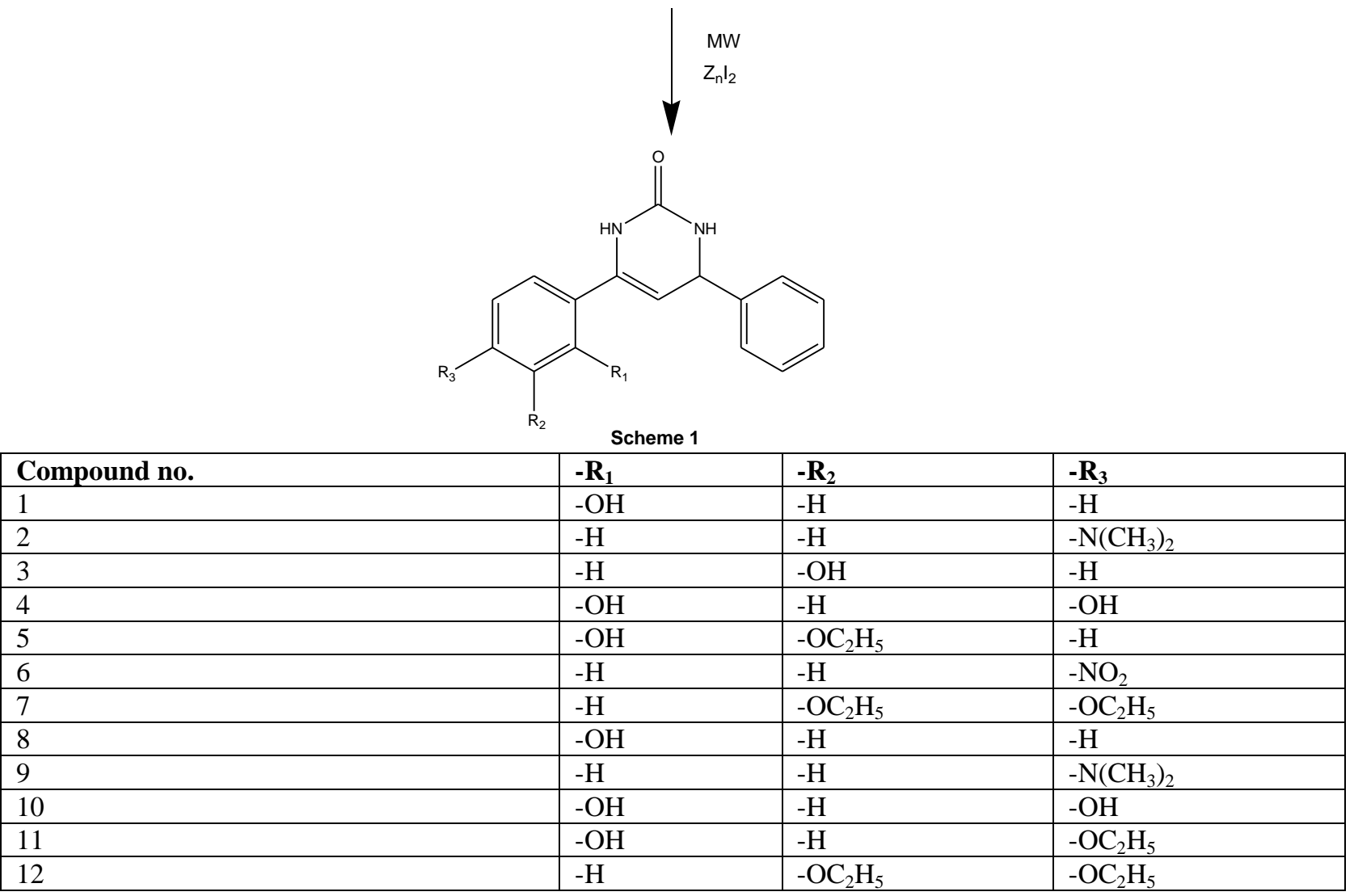

\section{Experimental:-}

All reactions were performed on a domestic microwave oven (Power $1200 \mathrm{~W}$ ). All reactants were obtained from commercial sources and freshly distilled prior to use. Melting points were taken in an electrically heated instrument and are uncorrected. Compounds were routinely checked for their purity on silica gel TLC plates and the spots were visualized by iodine vapors. IR spectra were recorded on Shimadzu 8201 PC FTIR spectrometer. PMR spectra were recorded on Bruker DRX $300 \mathrm{mhz}$ FT NMR spectrometer using TMS as internal reference and chemical shift values are expressed in $\delta$ units. Mass spectra were run on Jeol SX - 102spectrometer.

\section{General Procedure:-}

For the synthesis of compounds 1-12, a mixture of the appropriate aldehyde, acetophenone and urea or thiourea in equimolar concentration with a catalytic amount of $\mathrm{zni}_{2}$ in a $100 \mathrm{ml}$ glass tube was irradiated in a microwave oven in 
bursts of 15-20 seconds. The reaction was monitored by TLC. After the reaction was completed, distilled water was added into the flask and stirred for several minutes and then filtrated through a sintered funnel to afford crude product, which was further purified by recrystallization (etoh). Reaction details are given in Table 1.

Table 1:-Reaction Details.

\begin{tabular}{|l|l|l|l|l|l|}
\hline No. & Aldehyde & Amide & $\begin{array}{l}\text { Time } \\
(\text { Minutes })\end{array}$ & Yield (\%) & Mp $\left({ }^{0} \mathrm{C}\right)$ \\
\hline $\mathbf{1}$ & 2-hydroxybenzaldehyde & Urea & 6 & 65.5 & 250 \\
\hline $\mathbf{2}$ & 4-dimethylaminobenzaldehyde & Urea & 5 & 63.0 & 240 \\
\hline $\mathbf{3}$ & 3-hydroxybenzaldehyde & Urea & 6 & 75.2 & 232 \\
\hline $\mathbf{4}$ & 2,4-dihydroxybenzaldehyde & Urea & 6 & 55.0 & 265 \\
\hline $\mathbf{5}$ & 3-ethoxy,2-hydroxybenzaldehyde & Urea & 7 & 68.3 & 215 \\
\hline $\mathbf{6}$ & 4-nitrobenzaldehyde & Urea & 8 & 66.1 & 227 \\
\hline $\mathbf{7}$ & 3,4-diethoxybenzaldehyde & Urea & 5 & 70.0 & 260 \\
\hline $\mathbf{8}$ & 2-hydroxybenzaldehyde & Thiourea & 6 & 74.8 & 255 \\
\hline $\mathbf{9}$ & 4-dimethylaminobenzaldehyde & Thiourea & 6 & 65.0 & 242 \\
\hline $\mathbf{1 0}$ & 2,4-dihydroxybenzaldehyde & Thiourea & 5 & 67.0 & 234 \\
\hline $\mathbf{1 1}$ & 3-ethoxy,2-hydroxybenzaldehyde & Thiourea & 6 & 78.0 & 230 \\
\hline $\mathbf{1 2}$ & 2,3-diethoxybenzaldehyde & Thiourea & 5 & 76.0 & 222 \\
\hline
\end{tabular}

Table 2:-Spectral Data.

\begin{tabular}{|c|c|c|}
\hline & Mass $(\mathbf{m} / \mathbf{z})$ & 1H NMR Data $\left(\right.$ dmsod $\left._{6}\right)$ \\
\hline 1 & $266,250,190,174,150,98$ & $\begin{array}{l}\text { 7.06-7.14(m, 5H, arh }), 6.61-7.04(\mathrm{~m}, 4 \mathrm{H}, \operatorname{arh}), 6.0(\mathrm{~S}, 1 \mathrm{H}, \mathrm{N} \underline{\mathrm{H}}), 5.94(\mathrm{~s}, \\
1 \mathrm{H}, \mathrm{C} \underline{\mathrm{H}}) .\end{array}$ \\
\hline 2 & $293,250,217,174,98$ & $\begin{array}{l}\text { 7.06-7.14(m, 5H, arh }), 6.61-7.04(\mathrm{~m}, 4 \mathrm{H}, \operatorname{arh}), 6.0(\mathrm{~s}, 1 \mathrm{H}, \mathrm{N} \underline{\mathrm{H}}), 5.94(\mathrm{~s}, \\
1 \mathrm{H}, \mathrm{C} \underline{\mathrm{H}}), 4.87(\mathrm{~s}, 1 \mathrm{H}, \mathrm{C} \underline{\mathrm{H}}), 2.85\left(\mathrm{~s}, 3 \mathrm{H}, \mathrm{CH}_{3}\right) .\end{array}$ \\
\hline 3 & $266,250,198,174,98$ & $\begin{array}{l}\text { 7.06-7.14(m, 5H, arh }), 6.61-7.04(\mathrm{~m}, 4 \mathrm{H}, \operatorname{arh}), 6.0(\mathrm{~s}, 1 \mathrm{H}, \mathrm{N} \underline{\mathrm{H}}), 5.94(\mathrm{~s}, \\
1 \mathrm{H}, \mathrm{C} \underline{\mathrm{H}}), 4.87(\mathrm{~s}, 1 \mathrm{H}, \mathrm{C} \underline{\mathrm{H}}) .\end{array}$ \\
\hline 4 & $282,266,226,250,206,174,98$ & $\begin{array}{l}\text { 7.06-7.14(m, } 5 \mathrm{H}, \operatorname{arh}), 6.15-6.96(\mathrm{~m}, 3 \mathrm{H}, \operatorname{arh}), 6.5(\mathrm{~s}, 1 \mathrm{H}, \mathrm{C} \underline{\mathrm{H}}), 5.0(\mathrm{~s}, \mathrm{H} \text {, } \\
\text { aroh }), 6.0(\mathrm{~s}, 1 \mathrm{H}, \mathrm{N} \underline{\mathrm{H}}), 4.59(\mathrm{~s}, 1 \mathrm{H}, \mathrm{CH}) .\end{array}$ \\
\hline 5 & $310,294,266,250,234,174,98$ & $\begin{array}{l}\text { 7.06-7.14(m, 5H, arh }), 6.48-6.69(\mathrm{~m}, 3 \mathrm{H}, \operatorname{arh}), 6.0(\mathrm{~s}, 1 \mathrm{H}, \mathrm{NH}), 5.56(\mathrm{~s}, \\
1 \mathrm{H}, \mathrm{CH}), 5.0(\mathrm{~s}, \mathrm{H}, \operatorname{aroh}), 3.98\left(\mathrm{q}, 2 \mathrm{H}, \underline{\mathrm{C}}_{2}\right), 1.33\left(\mathrm{t}, 3 \mathrm{H}, \mathrm{CH}_{3}\right) .\end{array}$ \\
\hline 6 & $295,250,219,174,98$ & $\begin{array}{l}7.56-8.14(\mathrm{~m}, 4 \mathrm{H}, \mathrm{arh}), 7.06-7.14(\mathrm{~m}, 5 \overline{\mathrm{H}}, \operatorname{arh}), 6.23(\mathrm{~s}, 1 \mathrm{H}, \mathrm{C} \underline{\mathrm{H}}) \\
1 \mathrm{H}, \mathrm{NH}) .\end{array}$ \\
\hline 7 & $338,294,233,261174,98$ & $\begin{array}{l}\text { 7.06-7.14(m, 5H, arh }), 6.61-6.75(\mathrm{~m}, 3 \mathrm{H}, \operatorname{arh}), 6.0(\mathrm{~s}, 1 \mathrm{H}, \mathrm{N} \underline{\mathrm{H}}), 5.56(\mathrm{~s}, \\
1 \mathrm{H}, \mathrm{CH}), 3.98\left(\mathrm{q}, 2 \mathrm{H}, \mathrm{CH}_{2}\right), 1.33\left(\mathrm{t}, 3 \mathrm{H}, \mathrm{CH}_{3}\right) .\end{array}$ \\
\hline 8 & $282,266,206,190,114$ & $\begin{array}{l}\text { 7.06-7.14(m, } 5 \mathrm{H}, \operatorname{arh}), 6.77-7.13(\mathrm{~m}, 4 \mathrm{H}, \operatorname{arh}), 6.5(\mathrm{~s}, 1 \mathrm{H}, \mathrm{C} \underline{\mathrm{H}}), 5.0(\mathrm{~s}, 1 \mathrm{H} \text {, } \\
\text { aroh), } 2.0(\mathrm{~s}, 1 \mathrm{H}, \mathrm{N} \underline{\mathrm{H}}) .\end{array}$ \\
\hline 9 & $309,265,233,190,114$ & $\begin{array}{l}\text { 7.06-7.14(m, 5H, arh }), 6.54-7.12(\mathrm{~m}, 4 \mathrm{H}, \operatorname{arh}), 4.59(\mathrm{~s}, 1 \mathrm{H}, \mathrm{C} \underline{\mathrm{H}}), 2.85(\mathrm{~s}, \\
\left.3 \mathrm{H}, \mathrm{C}_{3}\right), 2.0(\mathrm{~s}, 1 \mathrm{H}, \mathrm{N} \underline{\mathrm{H}}) .\end{array}$ \\
\hline 10 & $298,282,266,222,206,114$ & $\begin{array}{l}\text { 7.06-7.14(m, 5H, arh }), 6.24-6.96(\mathrm{~m}, 3 \mathrm{H}, \operatorname{arh}), 6.5(\mathrm{~d}, 1 \mathrm{H}, \mathrm{CH}), 5.0(\mathrm{~s}, \\
1 \mathrm{H}, \operatorname{aroh}), 4.59(\mathrm{~d}, 1 \mathrm{H}, \mathrm{C} \underline{\mathrm{H}}), 2.0(\mathrm{~s}, 1 \mathrm{H}, \mathrm{N} \underline{\mathrm{H}}) .\end{array}$ \\
\hline 11 & $326,310,282,250,190,114$ & $\begin{array}{l}\text { 7.06-7.14(m, 5H, arh }), 6.48-6.69(\mathrm{~m}, 3 \mathrm{H}, \operatorname{arh}), 6.5(\mathrm{~d}, 1 \mathrm{H}, \mathrm{CH}), 5.0(\mathrm{~s}, \\
1 \mathrm{H}, \operatorname{aroh}), 3.98\left(\mathrm{q}, 2 \mathrm{H}, \mathrm{C}_{2}\right), 2.0(\mathrm{~s}, 1 \mathrm{H}, \mathrm{N} \underline{\mathrm{H}}), 1.33\left(\mathrm{t}, 3 \mathrm{H}, \mathrm{C}_{3}\right),\end{array}$ \\
\hline 12 & $352,325,310,282,278,190,114$ & $\begin{array}{l}\text { 7.06-7.14(m, } 5 \mathrm{H}, \operatorname{arh}), 6.61-6.75(\mathrm{~m}, 3 \mathrm{H}, \operatorname{arh}), 6.5(\mathrm{~d}, 1 \mathrm{H},=\mathrm{CH}), 5.56(\mathrm{~s}, \\
1 \mathrm{H}, \mathrm{C} \underline{\mathrm{H}}), 4.59(\mathrm{~d}, 1 \mathrm{H}, \mathrm{C} \underline{\mathrm{H}}), 3.98\left(\mathrm{q}, 2 \mathrm{H}, \mathrm{CH}_{2}\right), 2.0(\mathrm{~s}, 1 \mathrm{H}, \mathrm{N} \underline{\mathrm{H}}), 1.33(\mathrm{t}, 3 \mathrm{H}, \\
\left.\mathrm{CH}_{3}\right),\end{array}$ \\
\hline
\end{tabular}

Mass Spectral Analysis:-

Mass spectral studies of 3,4-dihydro-6-(2-hydroxyphenyl)-4-phenylpyrimidin-2(1H)-one has shown variation in fragmentation only due to differently substituted phenyl ring, hydroxyl group, ethoxy group, nitro group, dimethylamino group. All the compounds in general have exhibited a similar pattern of fragmentation (scheme 2). The mass and NMR spectral data of the compounds are given in Table 2. 


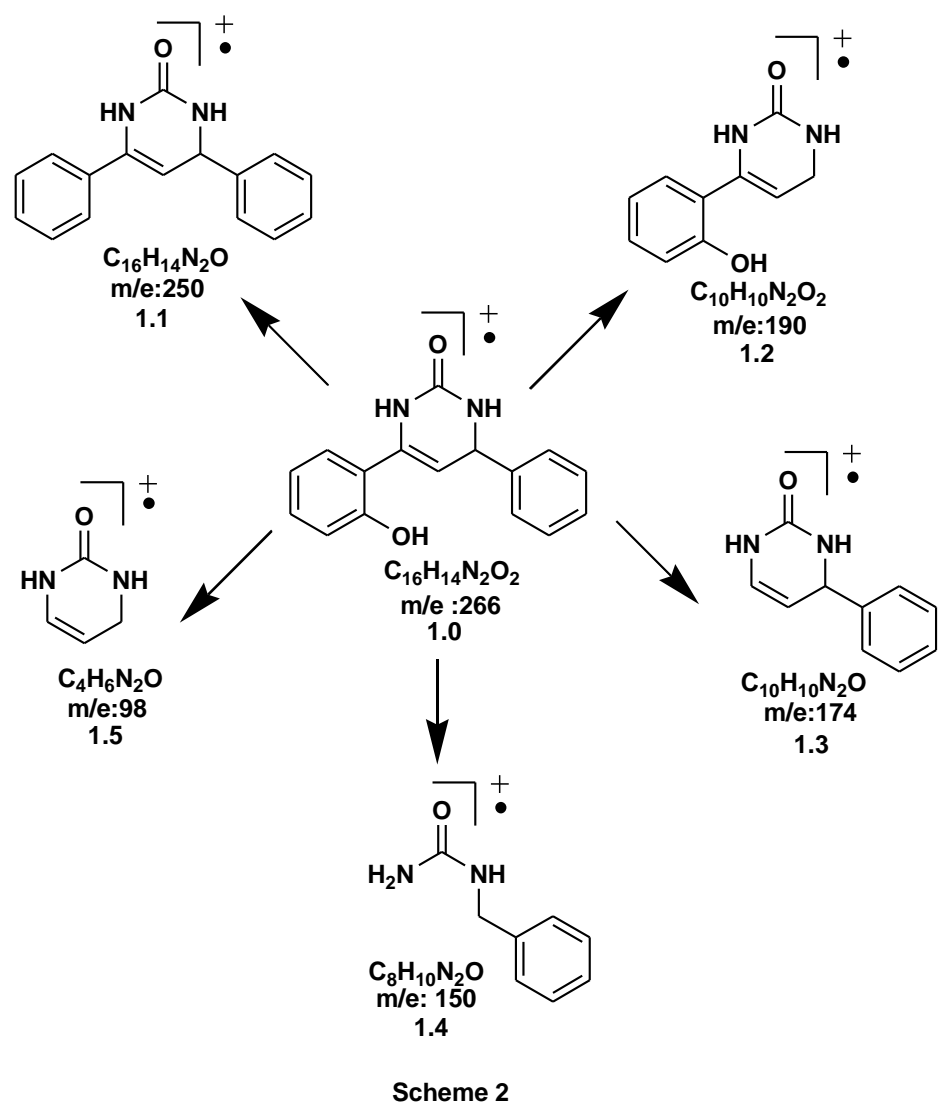

Biological activity:-

The compounds were tested for their anti-inflammatory activities against carrageenin induced rats paw edema using the method described ${ }^{6}$. The results are summarized in tables

Table 3:-Percentage edema growth relative to control at different time intervals (mean \pm SEM).

\begin{tabular}{|l|l|l|l|l|}
\hline Group & $\mathbf{0}(\mathbf{m i n})$ & $\mathbf{3 0}(\mathbf{m i n})$ & $\mathbf{9 0}(\mathbf{m i n})$ & $\mathbf{1 8 0}(\mathbf{m i n})$ \\
\hline Control & $100 \pm 0$ & $130.1 \pm 6.54(30.1)$ & $138.7 \pm 4.47(38.7)$ & $122.3 \pm 5.17(22.3)$ \\
\hline 1 & $100 \pm 0$ & $103.9 \pm 2.59(3.9)$ & $106.2 \pm 3.98(6.2)$ & $99.6 \pm 2.54(0.4)$ \\
\hline 2 & $100 \pm 0$ & $114.5 \pm 3.90(2.65)$ & $123 \pm 2.20(2.65)$ & $103 \pm 4.5(2.6)$ \\
\hline 3 & $100 \pm 0$ & $115.6 \pm 2.48(5.2)$ & $120 \pm 7.65(25.5)$ & $123 \pm 8.56(15.5)$ \\
\hline 4 & $100 \pm 0$ & $106.5 \pm 3.67(6.5)$ & $118.4 \pm 3.86(18.4)$ & $121.4 \pm 4.55(21.4)$ \\
\hline 5 & $100 \pm 0$ & $133.9 \pm 7.56(33.9)$ & $144.5 \pm 6.54(46.5)$ & $133.6 \pm 7.58(33.6)$ \\
\hline 6 & $100 \pm 0$ & $118.9 \pm 3.56(23.9)$ & $124.5 \pm 5.54(6.2)$ & $123.6 \pm 7.58(30.6)$ \\
\hline 7 & $100 \pm 0$ & $108.1 \pm 7 . .05(44.2)$ & $114.8 \pm 6.80(14.5)$ & $123.8 \pm 3.20(23.6)$ \\
\hline 8 & $100 \pm 0$ & $124.9 \pm 2.25(23.2)$ & $134.9 \pm 6.50(16.4)$ & $143.3 \pm 8.20(25.7)$ \\
\hline 9 & $100 \pm 0$ & $116.3 \pm 2.30(14.3)$ & $123.3 \pm 4.32(23.2)$ & $132.2 \pm 6.36(14.8)$ \\
\hline 10 & $100 \pm 0$ & $126.8 \pm 3.56(14.8)$ & $127.7 \pm 3.33(26.6)$ & $130.1 \pm 1.30(42.3)$ \\
\hline 11 & $100 \pm 0$ & $113.5 \pm 2.20(18.2)$ & $131.5 \pm 5.50(15.5)$ & $136.0 \pm 5.30(16.4)$ \\
\hline 12 & $100 \pm 0$ & $123.5 \pm 3.50(15.6)$ & $134.6 \pm 8.56(18.5)$ & $141.7 \pm 7.23(31.2)$ \\
\hline Ibuprofen & $100 \pm 0$ & $101.7 \pm 2.11$ & $108 \pm 3.27$ & $11.6 \pm 4.19$ \\
\hline
\end{tabular}

Table 4:-Anti-inflammatory activity screening data of the compounds.

\begin{tabular}{|c|c|c|}
\hline Compound No. & Mean Difference & Percent Activity $(\mathbf{1 0 0 m g} / \mathbf{K g})$ \\
\hline $\mathbf{1}$ & 35.20 & 59 \\
\hline $\mathbf{2}$ & 26.14 & 36 \\
\hline $\mathbf{3}$ & 25.63 & 35 \\
\hline $\mathbf{4}$ & 25.63 & 37 \\
\hline
\end{tabular}




\begin{tabular}{|l|l|l|}
\hline $\mathbf{5}$ & 24.35 & 39 \\
\hline $\mathbf{6}$ & 23.18 & 40 \\
\hline $\mathbf{7}$ & 17.73 & 49 \\
\hline $\mathbf{8}$ & 32.50 & 52 \\
\hline $\mathbf{9}$ & 23.18 & 52 \\
\hline $\mathbf{1 0}$ & 23.50 & 35 \\
\hline $\mathbf{1 1}$ & 21.19 & 32 \\
\hline $\mathbf{1 2}$ & 22.01 & 45 \\
\hline
\end{tabular}

\section{Acknowledgement:-}

The authors acknowledge the help of RSCI, CDRI Lucknow for providing the spectral analysis and biological screening.

\section{References:-}

1. Biginelli, P. Chem. Ber.1891, 24, 1317, 2962.

2. Pechman, H.; Duisberg, C. Chem. Ber. 1883, 16, 2119.

3. Bose, A, K.; Pednekar, S,;Ganguly, S, N,; Chakraborty, G,; Manhas, M, S Tetrahedron Lett. $2004,45,8351$.

4. Wang, Z.; Xu, L.; Xia, C.; Wang, H. Tetrahedron Lett. 2004, 45, 7951-7953.

5. Biginelli, P. Gazz. Chim. Ital. 1893, 23, 360-362.

6. Kappe,C.O.; Kumar, D.; Varma, R.S. Synthesis 1999, 10, 1799-1803;

7. Ashish Kumar Tewari, RashmiDubey, Anil Mishra, Med Chem Res, 2010 DOI 10.1007/s00044-009-9290-9. 